NASA Contractor Report 182297

AIAA-89-1690

\title{
Unsteady Stagnation-Point Heat Transfer During Passage of a Concentrated Vortex
}

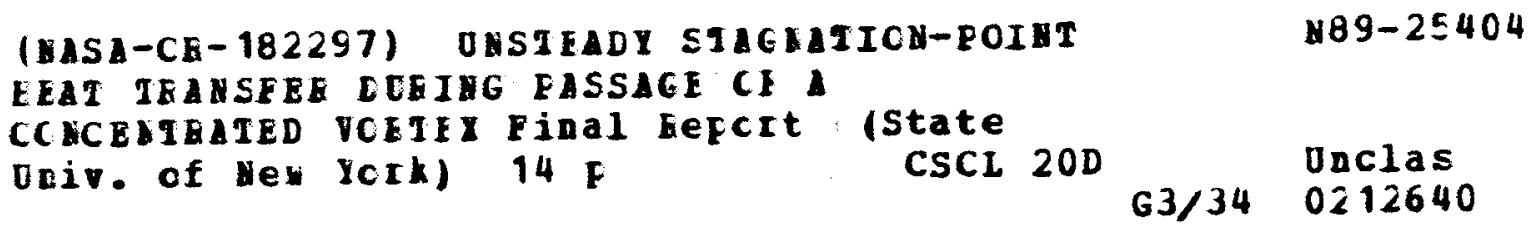

David L. Rigby and William J. Rae

State University of New York at Buffalo

Buffalo, New York

May 1989

Prepared for

Lewis Research Center

Under Grant NGT-33-183-805

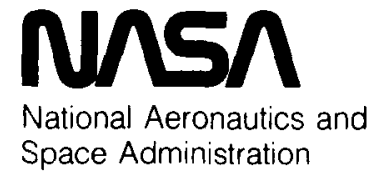

Space Administration 


\section{UNSTEADY STAGNATION-POINT HEAT TRANSFER DURING PASSAGE OF A CONCENTRATED VORTEX*}

David L. Rigby** and William J. Rae***

Department of Mechanical and Aerospace Engineering

State University of New York at Buffalo

Buffalo, NY 14260

\section{Abstract}

The unsteady boundary layer due to a single rectilinear vortex filament approaching a twodimensional stagnation point is investigated. Assuming the vortex remains far from the surface, incompressible potential-flow theory is used to determine the time-dependent inviscid flow field. The unsteady boundary-layer equations are solved by an alternating-direction-implicit finitedifference method. Two mechanisms which cause fluctuations in heat transfer are the unsteady velocity field in the boundary layer and secondly, the unsteady total temperature at the edge of the boundary layer. The relative importance of these mechanisms is dependent upon the total temperature fluctuations relative to the imposed temperature difference. As a vortex approaches a stagnation point it may be forced to one side of the stagnation line or the other, depending on its initial position. Results are presented for both of these cases.

\section{Introduction}

High-frequency heat-transfer fluctuations are a source of concern in a number of gas-turbine flow environments, since the thermal stresses which they cause can limit fatigue life even when the time-averaged level of heat transfer is well within design limits. The Space Shuttle Main Engine (SSME) turbopumps are one instance where this phenomenon has received attention, as part of the SSME Durability Program. The research reported in the present paper is part of an experimental/analytical effort that has been supported by the Durability Program, at the Calspan-UB Research Center (CUBRC) $1-3$.

In the experimental portions of the latter effort, time-resolved measurements of the distributions of heat-transfer rate have been made in a shock-tunnel facility, using full-scale turbine hardware operating under rated conditions. The data come from thin-film heat-transfer gages, which allow microsecond time-scale and millimeter spatial-scale resolution.

The data taken in the experimental part of the effort display a very rich frequency content, including a number of very sharp 'spikes' which rise to factors as large as two above the mean level, and drop to values near zero, over an interval on the order of microseconds. The application of phase averaging to these data tends to remove most of this 'spikiness', allowing the fundamental blade-to-blade periodicity (with

*Supported by the Graduate Student Researchers Program at the NASA Lewis Research Center. This work was directed at NASA Lewis by Dr. Louis A. Povinelli. periods on the order of 100 microseconds) to stand out. The interpretation of this fundamental unsteadiness in terms of laminar/turbulent transition triggered by wake cutting has been pursued in other papers from this program ${ }^{4}, 5$.

However, the question remains as to the source of the higher-frequency spikes, especially in regard to their property of displaying heattransfer levels well below the mean. The lower limits of these excursions are difficult to explain in terms of fluctuations in the thermal boundary-layer thickness; these might account for heat-transfer reductions on the order of a factor of two due to thickening of the thermal layer, but levels approaching zero exceed the bounds of this mechanism.

An alternate explanation that may account for these down-going spikes is the passage of a concentrated vortex over the blade leading edge. These vortices, shed from the trailing edges of an upstream stator row, have decayed only slightly by the time they are intercepted by a downstream rotor. A number of recent studies have described the very rapid velocity-boundary-layer changes that occur during the close approach of a vortex to the surface 6 . In addition, the flow unsteadiness causes a momentary redistribution of stagnation temperature ${ }^{9}$. The present work was undertaken in an attempt to clarify whether the combined effects of veloc1ty and temperature unsteadiness during vortex passage over a leading edge might explain the spikes seen in the experimental program.

The net result of the research is an affirmative answer to this question; it does appear that the combined effects of the fluctuations in velocity and temperature induced by the motion of the vortex produce a heattransfer 'signature' that is qualitatively similar to that seen in the experiments.

The analysis that leads to this conclusion invokes a number of simplifying assumptions, in an effort to gain as much analytical understanding as possible. It assumes a low mach-number inviscid flow, makes use of incompressible potential-flow theory as a source of the time-dependent vortexinduced portions of the flow-field, and employs laminar, noninteracting boundary-layer theory to describe the viscous layer. The geometry considered is a two-dimensional stagnation point; thus the baseline flow and heat transfer that occur long before passage of the vortex are given by the classical solutions of Hiemenz and Frossling 11 . When the vortex comes near the surface, it speeds up considerably; thus the timedependent contributions to the velocity and temperature fields become pronounced. 
The unsteady boundary-1ayer equations are solved by an alternating-direction-implicit method ${ }^{72}$, for a prescribed vortex trajectory. The temperature at the boundary-layer edge is allowed to have a constant level different from the wall temperature, and in addition to undergo the fluctuation caused by energy segregation during the vortex passage. At this level of approximation, the velocity and temperature fields are uncoupled, and the temperature in the boundary layer can be expressed as the sum of two terms, corresponding to the imposed temperature difference plus the induced fluctuation. The resulting heat-transfer rate is the sum of two corresponding terms. It is clear that very low heat-transfer levels with very short duration are possible with certain combinations of the amplitudes of the two temperature fields.

\section{Formulation}

Consider a steady two-dimensional stagnationpoint flow; let the flow be directed toward a wall located at the plane $\bar{y}=0$, with the stagnation point at $\bar{x}=\bar{y}=0$, see Figure 1 . The overbar is used to denote dimensional quantities. The temperature at the wall, $\overline{\mathrm{T}}_{\mathrm{w}}$, as well as the stagnation temperature far from the wall, $\overline{\mathrm{T}}_{0}$, will be constant.

Now, in addition, let a single inviscid twodimensional vortex with strength, $\bar{\Gamma}$, approach from upstream infinity. A vortex Reynolds number may be defined as $\operatorname{Re}_{\mathrm{v}}=\frac{\bar{\Gamma}}{2 \pi \bar{\nu}}$, where $\bar{\nu}$ is the kinematic viscosity. It is assumed that $\operatorname{Re}_{\mathrm{v}}$ is large so that the vortex can affect the thin viscous region near the wall while remaining in the effectively inviscid region far from the wall.

The motion of the vortex and unsteady velocities and temperature outside the boundary layer can be found using standard potential flow techniques 13 . The techniques actually used are those of classical incompressible flow, which may at first seem surprising, since they are being applied to a subsonic compressible-flow problem. The justification for this simplification is as follows: the temperature in the inviscid flow is governed by the compressible-flow energy equation:

$$
\bar{c}_{\mathrm{p}} \frac{\mathrm{DT}}{\overline{\mathrm{DT}}}=\frac{1}{\bar{\rho}} \frac{\mathrm{D} \overline{\mathrm{p}}}{\overline{\mathrm{D}}}
$$

The solution of this equation, for isentropic flow, is the compressible Bernoulli equation:

$$
\frac{\bar{T}}{\bar{T}_{\infty}}=1+\frac{Y-1}{2} M_{\infty}^{2}\left(1-\frac{\bar{v}^{2}}{\bar{u}_{\infty}^{2}}\right)-\frac{Y-1}{\bar{C}_{\infty}^{2}} \frac{\partial \bar{\phi}}{\partial \bar{t}}
$$

The middle term in this equation describes the adiabatic-compression mechanism of heating, while the third term is what accounts for the timedependent fluctuations in the external temperature. The former set tends to be spatially uniform in the vicinity of a stagnation point, whereas the latter shows significant spatial and temporal variations. (The middle term also contains a fluctuating part, in an unsteady flow).
The steady portion of the middle term is proportional to the square of the freestream Mach number, while the appropriate scale for the timedependent potential term is the square of the vortex strength divided by its distance from the surface. Since the latter scale is not affected, to first order, by the freestream Mach number, it is legitimate to approximate the vortex motion by incompressible-flow potential theory.

Because the distance of closest approach of the vortex is unknown in advance, there is no convenient length scale that can be used to define a dimensionless measure of the relative importance of time-dependent temperature fluctuations compared to those of steady adiabatic compression. The portion of the solutions below that reflect this unsteadiness will become large whenever the vortex appraaches sufficiently close to the surface. Of course, the distance of closest approach must be greater than that value where the viscous core and the boundary layer might interact; the cases shown below are far removed from this limit, so that the model of a potential vortex approaching a thin boundary layer can be used.

Introducing the following non-dimensional variables

$$
\begin{aligned}
& u=\bar{u} / \sqrt{\frac{\bar{A} \Gamma}{2 \pi}} ; v=\bar{v} / \sqrt{\overline{\nu A}} \\
& x=\bar{x} / \sqrt{\frac{\bar{\Gamma}}{2 \pi \bar{A}}} ; y=\bar{y} / \sqrt{\frac{\bar{\Gamma}}{2 \pi \bar{A}}} ; \eta=\bar{y} / \sqrt{\frac{\bar{\nu}}{\bar{A}}} \\
& t=\overline{A t} \\
& p=\left(\overline{\mathrm{p}} \cdot \overline{\mathrm{p}}_{\mathrm{o}}\right) /\left[\frac{\rho \mathrm{A} \Gamma}{2 \pi}\right] ; \theta=\frac{\overline{\mathrm{T}}-\overline{\mathrm{T}}_{\mathrm{w}}}{\overline{\mathrm{T}}_{\mathrm{o}}-\overline{\mathrm{T}}_{\mathrm{w}}}
\end{aligned}
$$

where $\bar{x}$ and $\bar{y}$ are coordinates tangent and normal to the surface respectively; also $\bar{t}$ is time, $\bar{p}$ is the pressure, $\overline{\mathrm{T}}$ is the temperature and $\bar{u}$ and $\bar{v}$ are the tangent and normal velocities respectively. The constant $\bar{A}$ is the stagnation- point velocity gradient which determines the rate at which the velocity increases in the $\bar{x}$ direction; also the constants $\bar{p}_{0}$ and $\bar{T}_{0}$ are the steady total pressure and total temperature, respectively.

The velocity of the vortex is

$$
\begin{aligned}
& \dot{x}_{v}(t)=x_{v}(t)+\frac{1}{2 y_{v}(t)} \\
& \dot{y}_{v}(t)=-y_{v}(t)
\end{aligned}
$$

It can be shown that if the vortex is located at $\left(\mathrm{x}_{\mathrm{vo}}, \mathrm{y}_{\mathrm{vo}}\right)$ at $\mathrm{t}=0$ then the position of the vortex is given by

$$
\begin{aligned}
& x_{v}(t)=\left[x_{v_{0}}+\frac{t}{2 y_{v_{0}}}\right] e^{t} \\
& y_{v}(t)=y_{v_{0}} e^{-t}
\end{aligned}
$$


The unsteady tangential velocity and the unsteady temperature induced at the wall are given by 14

$u_{e}(x, t)-x+\frac{2}{y_{v} R_{v}^{2}}$

$\theta_{e}(x, t)=1-E\left\{\frac{1}{2}\left[x+\frac{2}{y_{v} R_{v}}\right]^{2}+\frac{2\left(x-x_{v}\right)-2 \dot{x}_{v}}{y_{v} R_{v}^{2}}\right]$

also the total temperature is given by

$$
\theta_{\mathrm{e} 0}=1-E\left\{\frac{2\left(\mathrm{x}-\mathrm{x}_{\mathrm{v}}\right)-2 \dot{\mathrm{x}}_{\mathrm{v}}}{\mathrm{y}_{\mathrm{v}} \mathrm{R}_{\mathrm{v}}^{2}}\right\}
$$

where

$$
R_{v}^{2}(x, t)=1+\left[\frac{x-x_{v}}{y_{v}}\right]^{2}
$$

and

$$
E=\frac{\overline{A \Gamma}}{2 \pi c_{p}\left(\bar{T}_{o}-\bar{T}_{w}\right)}
$$

The importance of the bracketed term in equation (10) will depend on the value of $E$ and also on how large the terms within the bracket become during the passage of the vortex. To get an idea of an appropriate value for $E$, consider flow over a leading edge of radius $\bar{a}$ with freestream velocity $\bar{u}_{\infty}$. Then it can be shown that $\bar{A}=0\left[\frac{u_{\infty}}{\bar{a}}\right]$ and $\frac{\bar{\Gamma}}{2 \pi}=O\left(\bar{u}_{\infty} \bar{a} \lambda\right)$ where $\lambda$ is a constant on the order of unity (1.e. $\lambda-0.2)^{8}$. So

$$
E=0\left[\frac{\lambda \bar{u}_{\infty}^{2}}{c_{p}\left(\bar{T}_{0}-\bar{T}_{w}\right)}\right]=0\left(\lambda E_{c}\right)
$$

where $E_{c}=\frac{\bar{u}_{\infty}^{2}}{c_{p}\left(\bar{T}_{0}-\bar{T}_{\infty}\right)}$ is the Eckert number.

Notice this can also be written in terms of the mach number $M=\frac{\vec{u}_{\infty}}{\bar{c}_{\infty}}$ where for an ideal gas $\bar{c}_{\infty}=\sqrt{\gamma R T_{\infty}}, \gamma$ is the ratio of specific heats, $R$ is the gas constant. Then

$$
E=0\left[\frac{\lambda(\gamma-1)}{\left[1-\frac{T w}{T_{0}}\right]} \frac{M^{2}}{1+\frac{\gamma-1}{2} M^{2}}\right]
$$

To assume incompressible flow, as has been done in the present formulation, the double limit as $\mathrm{M}^{2} \rightarrow$ 0 and $\left[1-\frac{\overline{\mathrm{T}}_{\mathrm{w}}}{\overline{\mathrm{T}}_{0}}\right] \rightarrow 0$ must be taken.

From the above equation one can see that it is not inconsistent to choose a finite value of $\mathbf{E}$.

Since both $\lambda$ and $E_{c}$ are expected to have values somewhat less than one, values of $E$ which are quite small are investigated. Results are presented for $\mathrm{E}=0.0$ and $\mathrm{E}=0.02$.

Even for a small value of $E$, the bracketed term in equation (10) can be important during the passage of the vortex. The major effect of the vortex occurs directly beneath the vortex where $\left(x-x_{y}\right)$ is small, so in general the fluctuation in total temperature is directly proportional to the tangential $v \in l o c i t y$ of the vortex and inversely proportional to its distance from the surface. The non-dimensional boundary layer equations for mass, $x$-momentum, and energy are

$$
\begin{aligned}
& \frac{\partial u}{\partial x}+\frac{\partial v}{\partial \eta}=0 \\
& \frac{\partial u}{\partial t}+u \frac{\partial u}{\partial x}+v \frac{\partial u}{\partial \eta}=-\frac{\partial p}{\partial x}+\frac{\partial^{2} u}{\partial \eta^{2}} \\
& \frac{\partial \theta}{\partial t}+u \frac{\partial \theta}{\partial x}+v \frac{\partial \theta}{\partial \eta}=\frac{1}{\operatorname{Pr}} \frac{\partial^{2} \theta}{\partial \eta^{2}}+E\left\{\left[\frac{\partial u}{\partial \eta}\right]^{2}+\frac{\partial p}{\partial t}+u \frac{\partial p}{\partial x}\right\}
\end{aligned}
$$

where the pressure is known from the solution of the inviscid problem. Also, $\operatorname{Pr}$ is the Prandt number and $E$ is defined by equation (12). Equations (13-15) are subject to the following boundary conditions

$$
\begin{array}{lll}
\text { at } & \eta=0 & \mathrm{u}=\mathrm{v}=\theta=0 \\
\text { as } & \eta \rightarrow \infty & \mathrm{u} \rightarrow \mathrm{u}_{\mathrm{e}}, \quad \theta \rightarrow \theta_{\mathrm{e}}
\end{array}
$$

where $u_{e}$ and $\theta_{e}$ are given by equations (8) and (9) respectively.

To facilitate the numerical solution of equations (13) and (14) an unsteady stream function is defined by

$\mathrm{u}=\frac{\partial \psi}{\partial \eta} \quad ; \quad \mathrm{v}=-\frac{\partial \psi}{\partial \mathrm{x}}$

It may be shown that equations (17) satisfy the continuity equation identically and the $\mathrm{x}$-momentum equation can be written

$\frac{\partial u}{\partial t}+u \frac{\partial u}{\partial x}-\frac{\partial \psi}{\partial x} \frac{\partial u}{\partial \eta}=-\frac{\partial p}{\partial x}+\frac{\partial^{2} u}{\partial \eta^{2}}$

with the following boundary conditions

$$
\begin{array}{lll}
\text { at } & \eta=0 & \psi=\mathrm{u}=0 \\
\text { as } & \eta \rightarrow \infty & \mathrm{u} \rightarrow \mathrm{u}_{\mathrm{e}}
\end{array}
$$

The freestream velocity is often included in the definition of the stream function to simplify the boundary condition. In the present case this is not convenient since the unsteadiness of $u_{e}$ will cause it to vanish at $x$-positions where the velocity profile is not identically zero.

Owing to the linearity of the energy equation (15), it is possible to define two new temperature fields such that

$\theta=\theta_{1}+\mathrm{E} \theta_{2}$

where $\theta_{1}$ and $\theta_{2}$ must satisfy

$$
\begin{aligned}
& \frac{\partial \theta_{1}}{\partial t}+u \frac{\partial \theta_{1}}{\partial \mathrm{x}}+\mathrm{v} \frac{\partial \theta_{1}}{\partial \eta}=\frac{1}{\operatorname{Pr}} \frac{\partial^{2} \theta_{1}}{\partial \eta^{2}} \\
& \frac{\partial \theta_{2}}{\partial t}+u \frac{\partial \theta_{2}}{\partial \mathrm{x}}+\mathrm{v} \frac{\partial \theta_{2}}{\partial \eta}=\frac{1}{\operatorname{Pr}} \frac{\partial^{2} \theta_{2}}{\partial \eta^{2}}+\left[\frac{\partial u}{\partial \eta}\right]^{2}+\frac{\partial p}{\partial t}+u \frac{\partial p}{\partial x}
\end{aligned}
$$


subject to the boundary conditions

$$
\begin{array}{lll}
\text { at } & \quad \eta=0 & \theta_{1}=\theta_{2}=0 \\
\text { as } & \eta \rightarrow \infty & \theta_{1} \rightarrow 1, \theta_{2}+\frac{\theta_{\mathrm{e}}-1}{\mathrm{E}}
\end{array}
$$

Notice that a parametric investigation of the effect of $E$ on $\theta$ requires solving for $\theta_{1}$ and $\theta_{2}$ only once.

\section{Initial Condition}

To begin the numerical calculation the solution over the entire $(x, y)$ plane must be known at the initial time t=o. It is possible to find an asymptotic solution, valid when the vortex is far from the wall, which can be used for the initial condition, as follows:

Expanding in powers of $\left(1 / y_{v}\right)$ the inviscid results for $u_{e}$ and $\theta_{e}$ become

$u_{e}(x, t)=x+\frac{2}{y_{v}}+o\left[\frac{1}{y_{v}^{3}}\right]$

$\theta_{e}(x, t)=1-E\left\{\frac{1}{2} x^{2}+\frac{4 x}{y_{v}}\right\}+0\left[\frac{x_{v}}{y_{v}}, \frac{1}{y_{v}^{2}}\right]$

also

$\frac{\partial p}{\partial x}=-x-\frac{4}{y_{v}}+0\left[\frac{1}{y_{v}^{3}}\right]$

$\frac{\partial p}{\partial t}=-\frac{4 x}{y_{v}}+0\left[\frac{x_{v}}{y_{v}} \frac{1}{y_{v}^{2}}\right]$

By expanding the unsteady stream function and temperatures as

$\psi(\mathrm{x}, \eta, t)=\mathrm{x} \psi_{\mathrm{o}}(\eta)+\frac{2}{\mathrm{y}_{\mathrm{v}}} \psi_{1}(\eta)+o\left(\frac{1}{\mathrm{y}_{\mathrm{v}}}\right)$

$\theta_{1}(\mathrm{x}, \eta, \mathrm{t})=\theta_{10}(\eta)+\frac{1}{\mathrm{y}_{\mathrm{v}}} \theta_{11}(\eta)+o\left(\frac{1}{\mathrm{y}_{\mathrm{v}}}\right)$

$\theta_{2}(x, \eta, t)=-\frac{x^{2}}{2} \theta_{20}(\eta)+\frac{4 x}{y_{v}} \theta_{21}(\eta)+o\left[\frac{1}{y_{v}}\right]$

and introducing these relations into equations (18), (21) and (22) the following set of ordinary differential equations results

$\psi_{0}{ }^{2}-\psi_{0} \psi_{0}^{\prime \prime}=1+\psi_{0}^{\prime \prime \prime}$

$\left(1+\psi_{0}^{\prime}\right) \psi_{1}^{\prime}-\psi_{0} \psi_{1}^{\prime \prime}=2+\psi_{1}^{\prime \prime}$

$\frac{1}{\operatorname{Pr}} \theta i \dot{0}+\psi_{0} \theta \dot{i o}_{0}=0$

$$
\theta_{11}=0
$$

$\frac{1}{\operatorname{Pr}} \theta_{20}^{\prime}+\psi_{0} \theta_{20}^{\prime}-2 \psi_{0}^{\prime} \theta_{20}=2\left(\psi_{0}^{\prime}{ }^{2}-\psi_{0}^{\prime}\right)$

$\frac{1}{\operatorname{Pr}} \theta_{21}^{\prime}+\psi_{0} \theta_{21}^{\prime}-\left(1+\psi_{0}^{\prime}\right) \theta_{21}$

$$
=\psi_{0}^{\prime} \psi_{1}^{\prime}-\left(1+\psi_{0}^{\prime}\right)-\frac{1}{2} \psi_{1}^{\prime}\left(1-\theta_{20}\right)
$$

with boundary conditions

at $\eta=0 \quad \psi_{0}=\psi_{0}^{\prime}-\psi_{1}=\psi_{1}^{\prime}=\theta_{10}=\theta_{11}=\theta_{20}=\theta_{21}-0$

as $\eta \rightarrow \infty \quad \psi_{0}^{\prime}, \psi_{1}^{\prime}, \theta_{10}, \theta_{11}, \theta_{20}, \theta_{21} \rightarrow 1$

Note that equations (31) and (33) with the boundary conditions of equation (37) correspond 78 the steady stagnation-point problems of Hiemenz and Frossing ${ }^{15}$. Each of equations (31)-(36) together with boundary conditions (37) are solved numerically using methods which are second-order accurate.

Assuming the initial vortex position is chosen large enough, equations (28)-(30) will produce an accurate initial condition for the problem.

\section{Numerical Method}

The numerical method used to solve the boundary-layer problem described by equations (18), (21) and (22) is similar to that described by Peridier and Walker $(1988)^{7}, 12$. The method is a factored alternating-difference-implicit method (ADI) which uses upwind-downwind differencing for the first-order derivatives. The following is a brief description of the method. For a more complete description see Peridier and Walker $(1988)^{7}, 12$.

A rectangular grid is defined, where the indices $i, j, n$ denote the $x$-direction, the $\eta$ direction and time, respectively. Constant grid spacing is used, where $\Delta x, \Delta \eta$ and $\Delta t$ denote the respective step sizes. Consider in general the following equation

$\frac{\partial F}{\partial t}=\mathrm{T} \frac{\partial^{2} F}{\partial \eta^{2}}+\mathrm{P} \frac{\partial \mathrm{F}}{\partial \eta}+\mathrm{Q} \frac{\partial \mathrm{F}}{\partial \mathrm{x}}+\mathrm{M}$

where Table 1 shows how this equation can represent each of equations (18), (21) and (22). A finite-difference approximation to equation (38) is written at the point $(i, j, n+1 / 2)$. The coefficients are approximated using a simple average, for example

$\bar{P}_{i j}=\frac{1}{2}\left[P_{i j}^{n+1}+P_{i, j}^{n}\right]$

where the overbar here denotes that averaging has been done. The derivatives are also averaged in this manner. The second derivative is approximated by a central difference. The first derivative terms are approximated using an upwind-downwind scheme according to the sign of the coefficient. For example

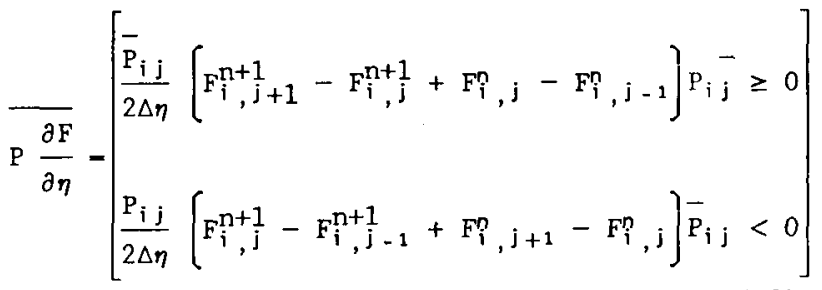


Equation 40 may be written more compact1y by defining the operators $\mathrm{x}_{\eta}^{+}$and $\mathrm{x}_{\eta}^{-}$as

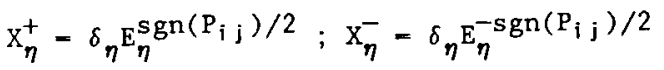

where $\delta_{\eta}$ is the central difference operator

$\delta_{\eta} F_{i, j}^{n}=F_{i, j+1 / 2}^{n}-F_{i}^{n}, j-1 / 2$

and $E_{\eta}^{ \pm 1 / 2}$ is defined by

$E_{\eta}^{ \pm 1 / 2} F_{i, j}^{n}=F_{i}^{n}, j \pm 1 / 2$

It follows that equation (40) can be written

$\overline{P \frac{\partial F}{\partial \eta}}=\frac{\bar{P}_{i j}}{2 \Delta \eta}\left[X_{\eta}^{+} F_{i, j}^{n+1}+X_{\eta}^{-} F_{i}^{n}, j\right]$

Similarly by defining

$X_{x}^{+}=\delta_{x} E^{\operatorname{sgn}\left(\bar{Q}_{i j}\right) / 2} ; X_{x}^{-}=\delta_{x} E^{-\operatorname{sgn}\left(\bar{Q}_{i j}\right) / 2}$

$\delta_{x} F_{i j}^{n}=F_{i+1 / 2, j}^{n}-F_{i-1 / 2, j}^{n}$

$E_{x}^{ \pm 1 / 2} F_{i, j}^{n}=F_{i \pm 1 / 2, j}^{n}$

the approximation to $\overline{Q \frac{\partial F}{\partial x}}$ can be written

$\overline{Q \frac{\partial F}{\partial x}}=\frac{\bar{Q}_{i j}}{2 \Delta x}\left[X_{x}^{+} F^{n+1} i, j+X_{x}^{-} F_{1, j}^{n}\right]$

The finite difference approximation to equation (38), written in factored form is

$\tilde{F}_{i, j}-\frac{\Delta t}{2 \Delta x}\left[\bar{Q}_{i, j} X_{x}^{+}\right] \tilde{F}_{i, j}-D_{i, j}$

$F_{i}^{n+1}-\frac{\Delta t}{2 \Delta \eta^{2}}\left[\bar{T}_{i, j} \delta_{\eta}^{2}+\Delta \eta \bar{P}_{i j} X_{\eta}^{+}\right] F_{i, j}^{n+1}=\tilde{F}_{i, j}$

where

$D_{i, j}=F_{i, j}^{n}+\frac{\Delta t}{2}\left[T_{i, j} \frac{\delta \eta^{2}}{\Delta \eta_{2}}+\bar{P}_{i, j} \frac{x \bar{\eta}}{\Delta \eta}+\bar{Q}_{i, j} \frac{X_{x}^{-}}{\Delta x}\right] F_{i, j}^{n}$

$+\Delta t \bar{M}_{i, j}$

This method is second order accurate in space and time. The solution procedure is as follows. Equation (47) is solved along all lines of constant $\eta$. Then equation (48) is solved along all lines of constant $x$. The solution of equation (47), as well as equation (48), is accomplished using a direct method of solution for tridiagonal problems. The method involves a combination of cyclic reduction ${ }^{16}$ and the Thomas algorithm ${ }^{17}$. If equation (38) is nonlinear several iterations may be required to advance the solution in time. The coefficients are first estimated using previous time-plane values and then updated using values from the previous iterate. The procedure is repeated until the maximum difference between iterates is below a given tolerance. Notice that for a linear equation, as is the case for the energy equations (21), (22), the coefficients need not be iterated upon. However, as will be discussed below, the application of the downstream boundary condition may require iteration. To update the coefficients in the case of the momentum equation (18), the tangential velocity $u$ must be integrated to get $\psi$. This is done using Simpson's $1 / 3$ rule $^{18}$.

\section{Boundary Conditions}

Boundary conditions are applied around the entire perimeter of the numerical domain. At the surface, i.e., $\eta=0$

$$
\psi=u=\theta_{1}=\theta_{2}=0
$$

As $\eta \rightarrow \infty$ the solution must asymptotically approach the freestream values. The freestream boundary conditions are applied at $\eta-\eta_{\max }$ where $\eta_{\max }$ is chosen large enough so that increasing $\eta_{\max }$ does not affect the solution. A typical value of $\eta_{\max }$ is -9 .

The $x$-domain is $\left[-x_{\max }, x_{\max }\right]$ where $x_{\max }$ is chosen large enough so that increasing $x_{\max }$ does not affect the solution. The effect of the vortex varies inversely with the distance from the vortex so the boundaries of the $x$-domain are not expected to experience much unsteadiness. This fact makes it possible to use a first-order-accurate extrapolation at the $x$-boundary without introducing any appreciable error in the region near the vortex. The method is developed by writing a Taylor series expansion about the grid point just inside the boundary. For example, if $x(1)=-x_{\max }, x(2)=-x_{\max }+\Delta x$ and so on, then

$\left.\left.F(1, j)=F(2, j)+\frac{\partial F}{\partial x}\right]_{i=2, j}(-\Delta x)+\frac{\partial^{2} F}{\partial x^{2}}\right]_{i=2, j} \frac{(-\Delta x)^{2}}{2}+\ldots$

The quantity $\left.\frac{\partial F}{\partial x}\right]_{i=2, j}$ is then approximated using values from the previous iteration.

$\left.\frac{\partial F}{\partial x}\right)_{i=2, j}-\frac{F^{\prime}(3, j)-F^{\prime}(1, j)}{2 \Delta x}+o\left(\Delta x^{2}\right)$

where the prime ()' denotes the previous iterates. Thus the boundary condition at $x=x_{\max }$ is

$F(1, j)-F(2, j)=-\frac{1}{2}\left[\left(F^{\prime}(3, j)-F^{\prime}(1, j)\right]+0\left(\Delta x^{2}\right)\right.$

Similarly at $\mathrm{x}=\mathrm{x}_{\max }$

$F(i \max , j)-F(i \max -1, j)=\frac{1}{2}\left[F^{\prime}(i \max , j)-F^{\prime}(i \max -2, j)\right]$

$$
+0\left(\Delta x^{2}\right)
$$

Notice that by applying the boundary conditions in this way more iterations may be required. Typically only 2 iterations are required for the endpoints to come within the tolerance. 
TABLE 1

\begin{tabular}{ccccccc}
\hline Equation & $\mathrm{F}$ & $\mathrm{T}$ & $\mathrm{P}$ & $\mathrm{Q}$ & $\mathrm{M}$ \\
\hline 18 & $\mathrm{u}$ & 1 & $-\frac{\partial \psi}{\partial \mathrm{x}}$ & $-\mathrm{u}$ & $-\frac{\partial \mathrm{p}}{\partial \mathrm{x}}$ \\
21 & $\theta_{1}$ & $1 / \mathrm{Pr}$ & $-\mathrm{v}$ & $-\mathrm{u}$ & 0 \\
22 & $\theta_{2}$ & $1 / \mathrm{Pr}$ & $-\mathrm{v}$ & $-\mathrm{u}$ & {$\left[\frac{\partial \mathrm{u}}{\partial \eta}\right]^{2}+\frac{\partial \mathrm{p}}{\partial t}+\mathrm{u} \frac{\partial \mathrm{p}}{\partial \mathrm{x}}$} \\
\hline
\end{tabular}

\section{Results}

It is possible to investigate all cases by choosing the sense of the vortex to be counterclockwise, since changing the sense of the vortex and the sign of $x_{v o}$ would result in a mirror image of the previous results. Two representative cases are presented. Each case is determined by the initial vortex position $\left(x_{v_{0}}, y_{y_{0}}\right)$. For each of the two cases heat transfer results are presented for $E=0.0$ and $\mathrm{E}=0.02$. The Prandt 1 number is held fixed and taken to be $\mathrm{Pr}=0.72$. Notice that since the vortex strength, $\bar{\Gamma}$, and the stagnation point gradient, $\bar{A}$, are included in the nondimensionalization they do not appear explicitly in the calculation.

For the first case the initial vortex position is $\left(\mathrm{x}_{\mathrm{yo}}, \mathrm{y}_{\mathrm{yo}}\right)-(0.025,20.0)$. This is referred to as the right case, since as the vortex approaches the surface it is pushed to the right of the geometric stagnation point, refer to Figure 2(a). In Figure 2(a) the vortex path is shown and three points are marked corresponding to $t=1.50$, 3.00 and 3.40 as shown. Results are then presented as functions of $x$ at these three times. As noted earlier, the sense of the vortex is taken to be counterclockwise. The vortex causes an increase in the external velocity, Figure 2(b), and its effect is felt mainly beneath the vortex. The resulting wall shear stress is shown in Figure 2(c). The wall shear stress increases beneath the vortex, but as the vortex comes nearer to the surface, the shear stress takes on values below the steady-state value in the region in front of the vortex (i.e. $x>x_{y}$ ). This occurs because the pressure gradient in this region is not as favorable as the steady value. At later times the pressure gradient would become adverse in front of the vortex and cause flow reversal, eventually causing a breakdown of the boundary-layer approximation. Figure 2(d) shows the displacement thickness as defined by

$$
\delta *=\frac{\overline{\delta^{*}}}{\sqrt{\frac{\bar{\nu}}{\bar{A}}}}=\int_{\eta=0}^{\infty}\left[1-\frac{\mathrm{u}}{\mathrm{u}_{\mathrm{e}}}\right] \mathrm{dy}
$$

There is a tendency for the boundary layer to thin behind the vortex and thicken in front of it. Note that near $x=0$ the displacement thickness fails to give meaningful information, since it is undefined when $u_{-}-0$. Plots of the streamfunction, Figure 3 , also give the indication of thinning behind and thickening in front of the vortex.
Figure 4 shows the external total temperature, the thermal boundary layer thickness, and the resulting heat transfer for $E=0.0$ and for $\mathrm{E}=0.02$. The thermal boundary layer thickness is defined by

$$
\delta_{\mathrm{T}}=\frac{\bar{\delta}_{\mathrm{T}}}{\sqrt{\frac{\bar{\nu}}{\mathrm{A}}}}=\left.\eta\right|_{\theta-.99 \theta_{\mathrm{e}}}
$$

Figure 4 (a) shows that the external total temperature for $E-0.02$ increases by as much as $30 \%$ below the vortex. For $E=0.0$ the external total temperature is identically equal to one. In Figure $4(\mathrm{~b}) \delta_{\mathrm{T}}$ is shown only for $\mathrm{E}-0.02$ because the differences between $E-0.00$ and $E-0.02$ are very slight. Note that as with the displacement thickness the trend is to thin behind the vortex and thicken in front of it.

Figure $4(c)$ shows heat transfer results for $E=0.0$ and $E=0.02$. The dimensionless heat transfer is defined by

$$
\left.\mathrm{q}_{\mathrm{w}}=\frac{\partial \theta}{\partial \eta}\right]_{\eta=0}=\frac{\overline{\mathrm{q}}_{\mathrm{w}}}{\left[\frac{-\overline{\mathrm{k}}\left(\overline{\mathrm{T}}_{\mathrm{o}}-\overline{\mathrm{T}}_{\mathrm{w}}\right)}{\sqrt{\frac{\bar{\nu}}{\bar{A}}}}\right.}
$$

where

$$
\left.\bar{q}_{w}=-\bar{k} \frac{\partial \bar{T}}{\partial \bar{y}}\right]_{y=0}
$$

Notice that for $E=0.0$ the heat transfer is higher where the boundary layer is thinner and vice versa. For $\mathrm{E}=0.02$ there is an additional increase in heat transfer due to the increased external total temperature. Figure 5 shows the heat transfer as a function of time for $x--2$, $-1,0,1,2$. From this it can be seen that the vortex passage has little effect on negative values of $x$ and even at $x=0$. It is interesting to note that a time average of the heat transfer would result in a net increase for this case. This is most easily seen at $x=2,0$. Since the time is non-dimensionalized by $A$, and $A$ may take on very large values, the time scale in Figure 5 is greatly exaggerated.

The second case corresponds to an initial vortex position of $\left(\mathrm{x}_{\mathrm{vo}}, \mathrm{y}_{\mathrm{yo}}\right)=(-.185,20.0)$ and will be referred to as the left case. Figure 6(a) shows the vortex path for this case. As in the right case results are presented as functions of $x$ for the three times corresponding to the points marked in Figure $6(a)$. For this case, the vortex is pushed toward the left (i.e. $x<0$ ) where the steady external velocity is negative, Figure $9(b)$. As in the right case the vortex causes a positive addition to the external velocity which drives it towards zero. When the vortex gets close enough a region of "inviscid flow reversal" occurs beneath the vortex. As can be seen in the plots of wall shear, Figure $6(\mathrm{c})$, and streamfunction, Figure 7 , a region of flow reversal forms beneath the 
vortex. This however, does not imply a breakdown of the boundary layer approximation since the flow is unsteady $(7,19)$. Note that the displacement thickness becomes singular where the outer velocity goes through zero and thus loses its usefulness for the present case. As a qualitative check on the boundary layer approximation, the maximum normal pressure gradient was calculated numerically from the results. This is done by using the $y$-momentum equation which was neglected in arriving at the result:

$$
-\frac{\partial \mathrm{p}}{\partial \eta}-\frac{\partial \mathrm{v}}{\partial t}+\mathrm{u} \frac{\partial \mathrm{v}}{\partial \mathrm{x}}+\mathrm{v} \frac{\partial \mathrm{v}}{\partial \eta}-\frac{\partial^{2} \mathrm{v}}{\partial \mathrm{y}^{2}}
$$

The right case which showed no signs of separation was found to have maximum normal pressure gradients comparable to the left case. Flow separation would be expected to occur eventually for either case.

Figure 8 shows the external total temperature, thermal boundary-layer thickness, and heat-transfer results for the left case. Note that the external total temperature (Figure 8a) is decreased as the vortex passes. The thermal boundary layer thickness (Figure 8b), behaves similar to the right case, i.e. thinning for $x<$ $x_{v}$ and thickening for $x>x_{v}$. Figure $8 c$ shows the resulting heat transfer. As expected it is higher where the thermal boundary layer has thinned and lower where the thermal boundary layer is thicker for $E=0.0$. For $E=0.02$ however, the heat transfer is lower than the $E=0.0$ result because of the drop in external total temperature. The time traces of heat transfer, Figure 9, show that values of $x>0$ and even $x=0$ are only slightly affected by the passage of the vortex. For this case there appears to be a net decrease in the heat transfer.

For each case the numerical domain was $0 \leq \eta$ $\leq 9,-6 \leq \mathrm{x} \leq 6$, with constant spatial grid sizes of $\Delta \eta=.125$ and $\Delta x=.05$. The time step was $\Delta t=.01$ for $t \leq 2.5$ and .005 for $t>2.5$. Each case took approximately 300 seconds of CPU time on a CRAY-XMP.

\section{Concluding Remarks}

The research described above grew out of a desire to find a mechanism for unsteady heattransfer fluctuations in a turbine rotor that lie both above and below the mean. The intuitive notion that the local heat-transfer rate is inversely proportional to the thermal-layer thickness is not adequate for explaining excursions that go well below the mean. Thus, the major focus of this effort was to determine whether the unsteady temperature fluctuations experienced during the passage of a vortex could produce heat-transfer fluctuations comparable to those of the mean flow. The results described above suggest an affirmative answer to this question: within the range of vortex trajectories and freestream conditions admitted by the approximations used, the heat-transfer levels from these two mechanisms are comparable, and the fluctuations are a significant fraction of the steady-state values.

It should be stressed that there are many features of the experiment that are not accounted for here, such as the complete rotor-blade geometry, the passage of multiple vortices, the effect of nonzero viscous core radii, and departures from two-dimensionality, to name just a few. Thus, it is not possible to make a direct comparison with the measurements. However, further study of these effects can now be carried out with confidence that the basic mechanism is a significant one in producing heat-transfer fluctuations in the stagnation region.

\section{Acknowledgment}

The authors gratefully acknowledge the support of this study by the Graduate Student Researchers Program at the NASA Lewis Research Center under grant number NGT33-183-805. This work was directed at NASA Lewis by Dr. Louis A. Povinelli. The authors are also grateful to Dr. Michael G. Dunn of Calspan Corporation for making time resolved heat transfer data available. With this data it was possible to get an idea of the nature of the unsteadiness within a gas turbine.

\section{References}

1. Dunn, M.G., George, W.K., Rae, W.J., Woodward, S.H., Moller, J.C. and Seymour, P.J., "Heat-Flux Meaurements for the Rotor of a Full-Stage Turbine: Part II, Description of Analysis Technique and Typical Time Resolved Measurements", ASME Paper 86-GT-78 (June 1986).

2. Rae, W.J., Taulbee, D.B., Civinskas, K.C., and Dunn, M.G., "Turbine Heat Transfer: Comparison of Short-Duration Measurements with State-Of-The-Art Predictions", AIAA Paper 86-1465 (June 1986).

3. Dunn, M.G., Seymour, P.J., Woodward, S.H., George, W.K. and Chupp, R.E., "Phase-Resolved Heat-Flux Measurements on the Blade of a FullScale Rotating Turbine", ASME Paper 88-GT-173 (June 1988)

4. Taulbee, D.B., Tran, L. and Dunn, M.G., "Stagnation Point and Surface Heat Transfer for a Turbine Stage: Prediction and Comparison with Data", ASME Paper No. 88-GT-30 (June 1988).

5. Taulbee, D.B. and Tran, L., "Prediction of Unsteady Rotor-Surface Heat Transfer from Wake Passings", AIAA Paper 89-1692 (June 1989)

6. Walker, J.D.A., "The Boundary Layer due to a Rectilinear Vortex", Proc. Roy. Soc. London, $\underline{A} 358$ (1978) $167-188$.

7. Peridier, V.J., Smith, F.T., and Walker, J.D.A., "Methods for the Calculation of Unsteady Separation", AIAA Paper 88-0604 (Jan 1988).

8. Bogucz, E.A., Dirik, E.A., and Lyman, F.A., "Unsteady Stagnation Point heat Transfer Due to the Motion of Free Stream Vortices", AIAA/ASME/SIAM/APS 1st National Fluid Dynamics Congress, Cincinnati, Ohio (July 25-28, 1988).

9. Kurosaka, M. et a1., "Energy Separation in a Vortex Street", J. Fluid Mech. 178, (1987) 1-29.

10. See Chapter V, Sec. 9 of H. Schlichting, Boundary-Layer Theory, 7th Edition, McGraw-Hill, New York (1979).

11. See Chapter XII, Sec. 3 of Ref. 10. 
12. Peridier, V. and Walker, J.D.A., "An Algorithm for Unsteady Flows with Strong Convection", NASA TM 100828, Oct. 1988.

13. Karamcheti, K., Principles of Ideal-Fluid Aerodynamics, Krieger Publishing Co. (1980).

14. Rigby, D.L., "An Investigation of Unsteady Stagnation - Point Heat Transfer", PhD Thesis, State University of New York at Buffalo (1989)

15. White, F.M. Viscous Fluid Flow, McGraw-Hill (1974).

16. Press, W.H., et al. Numerical Recipes, Ch. 17, Cambridge Univ. Press (1986)
17. Anderson, D. A., et a1., Computational Fluid Mechanics and Heat Transfer, Hemisphere Publishing Corp. (1984)

18. Curtiss, C.F. and Wheatley, P.O., "Applied and Numerical Analysis", Third Edition, Addison, Wesley, 1984.

19. Van Dommelen, L.L. and Shen, S.F. "The Genesis of Separation", Proc. Symp. Num. Phys. Aspects Aerodynamic Flows, T. Cebeci (Ed.) pp. 293-311, Springer-Verlag, 1982.1a

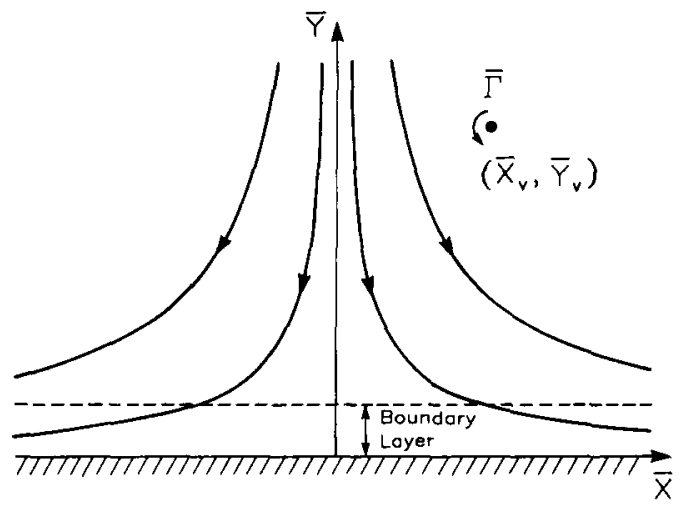

FIGURE 1. - GEOMETRY. 


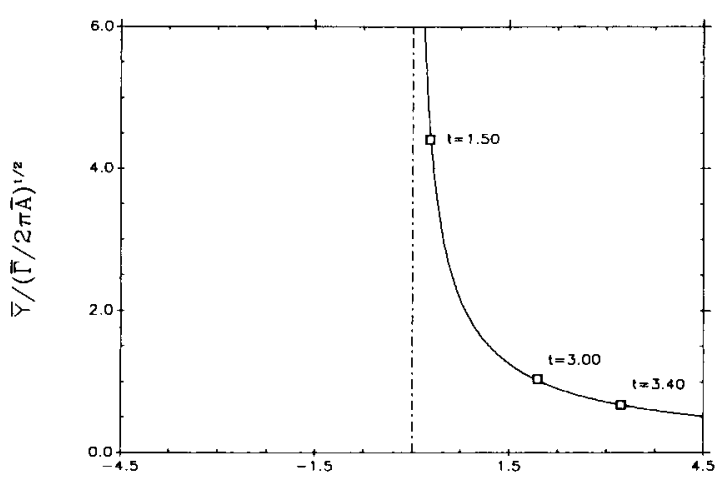

(a) VORTEX PATH.

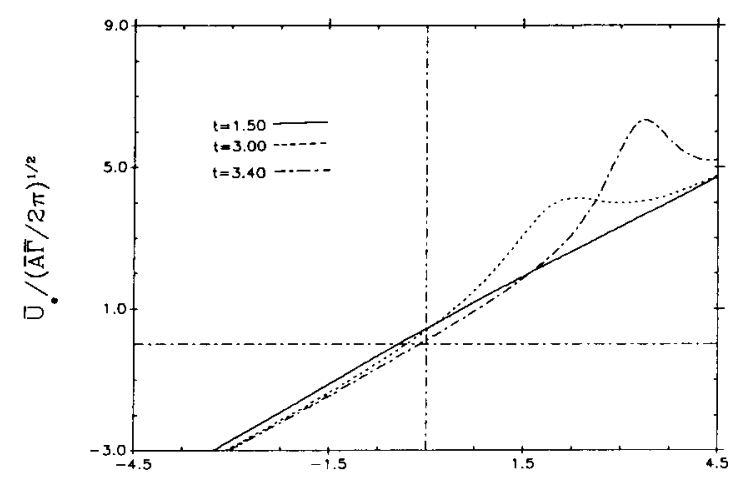

(b) EXTERNAL VELOCITY VERSUS $X$.

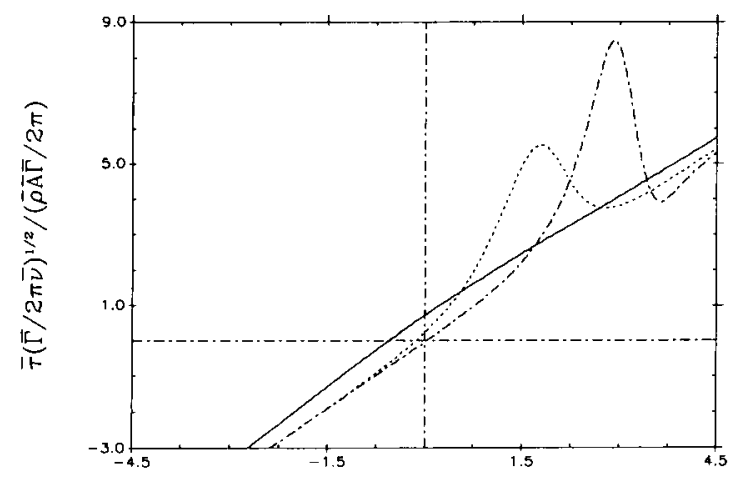

(c) WALL SHEAR VERSUS $X$.

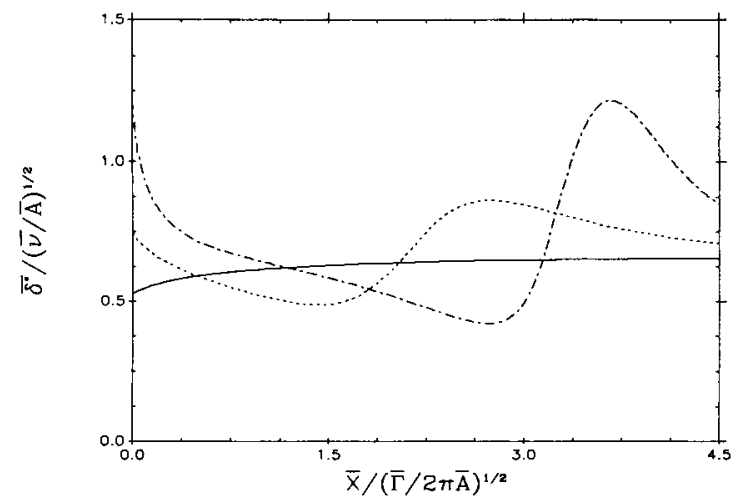

(d) DISPLACEMENT THICKNESS VERSUS $X$.

FIGURE 2. - MOMENTUM SOLUTION, RIGHT.

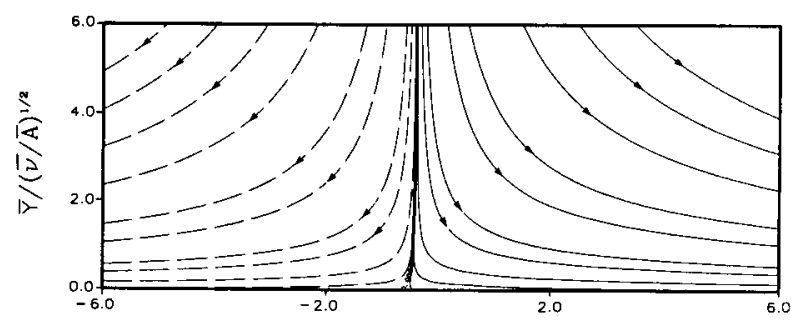

(a) $t=1.50$.
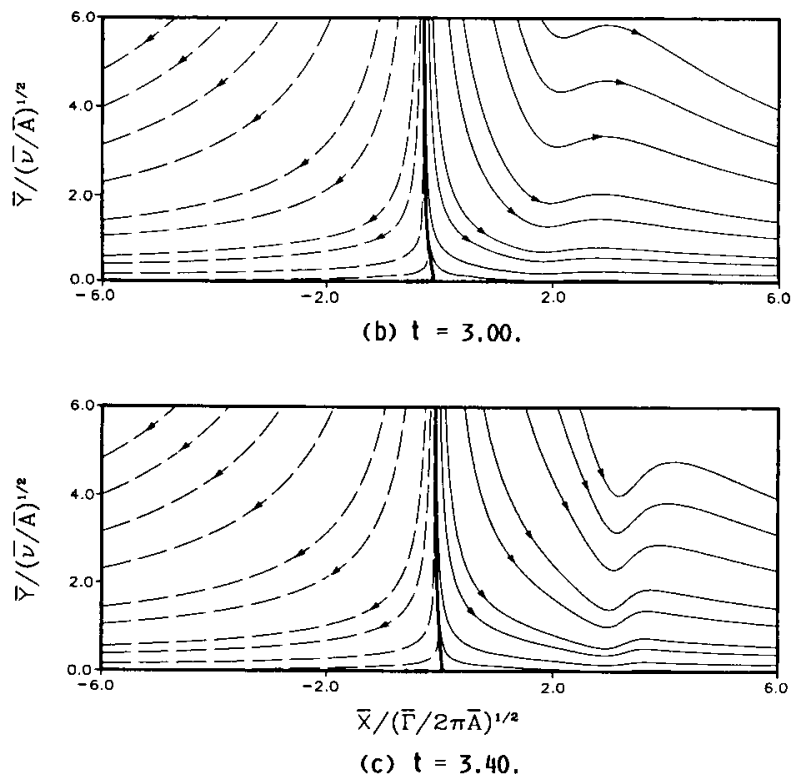

FIGURE 3. - STREAMLINES, RIGHT. 


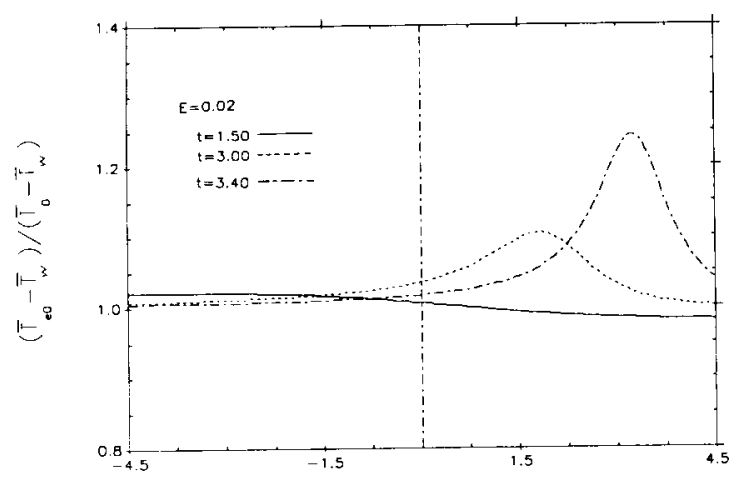

(a) EXTERNAL TOTAL TEMPERATURE VERSUS $X$.

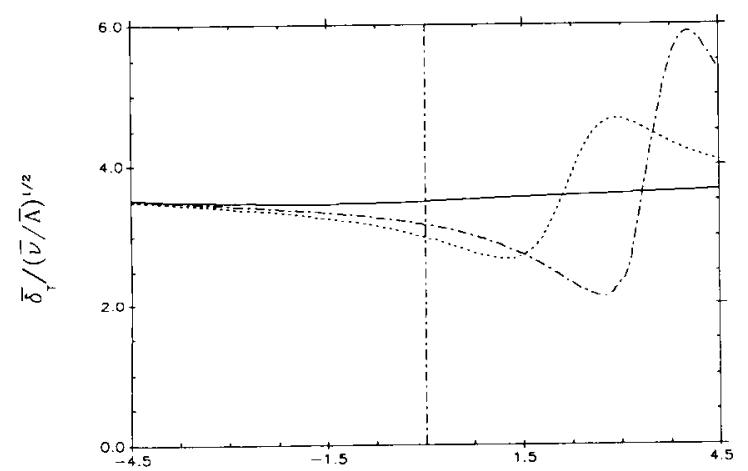

(b) DELTAT VERSUS $X$.

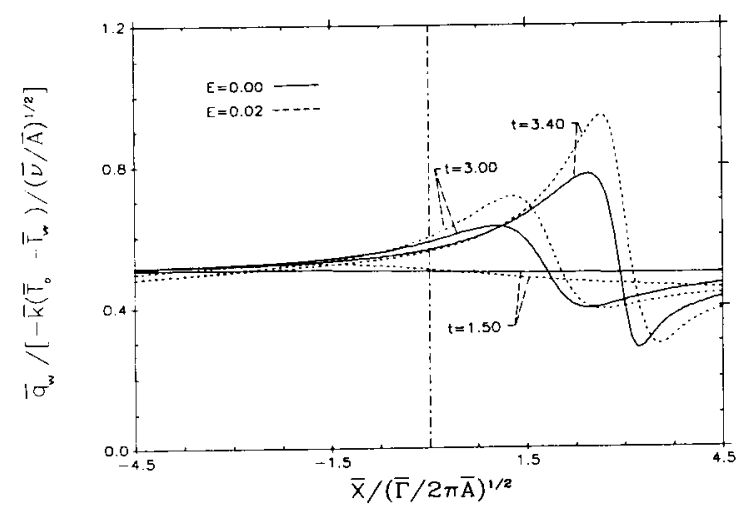

(c) WALL HEAT TRANSFER VERSUS $X$.

FIGURE 4. - ENERGY SOLUTION. RIGHT.

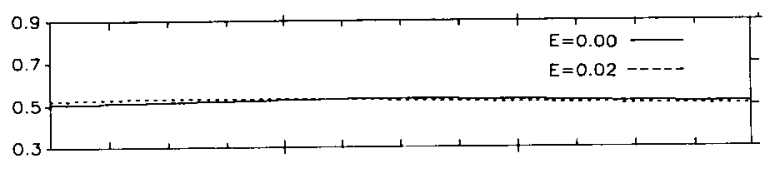

(a) $x=2.0$.

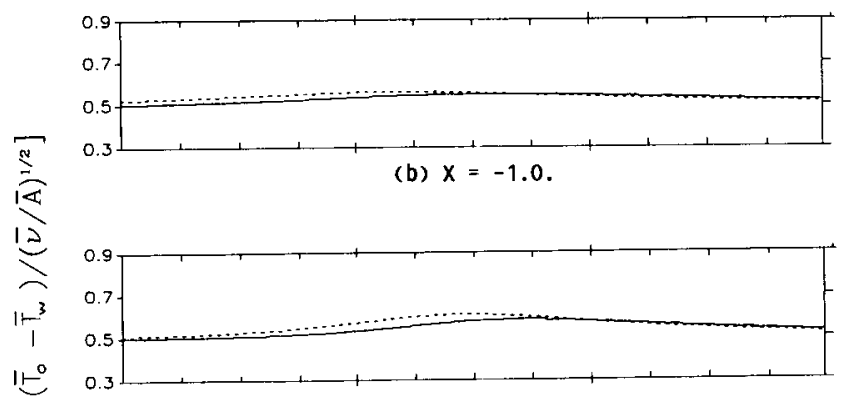

(c) $x=0.0$

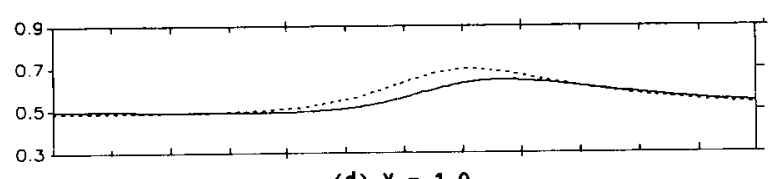

(d) $x=1.0$.

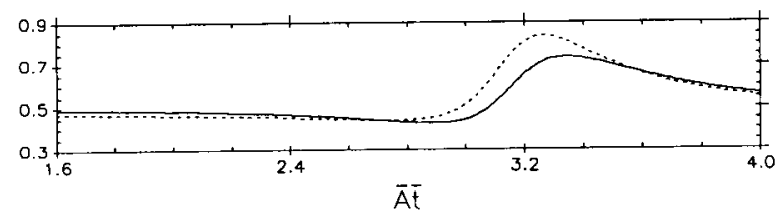

(e) $x=2.0$.

FIGURE 5. - WALL HEAT TRANSFER VERSUS $t$, RIGHT. 


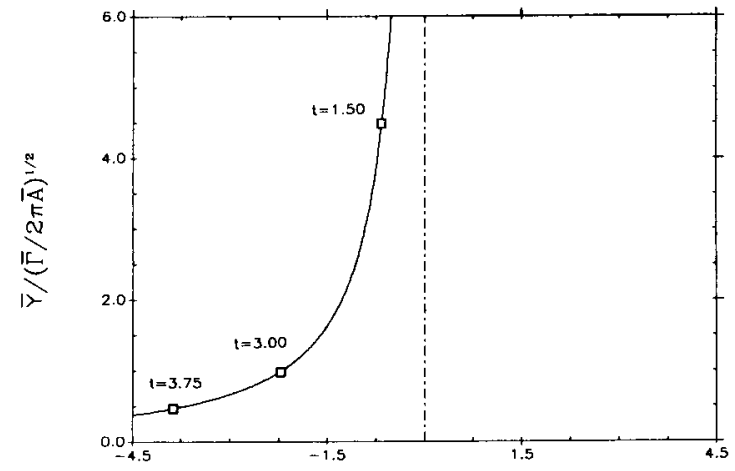

(a) VORTEX PATH.

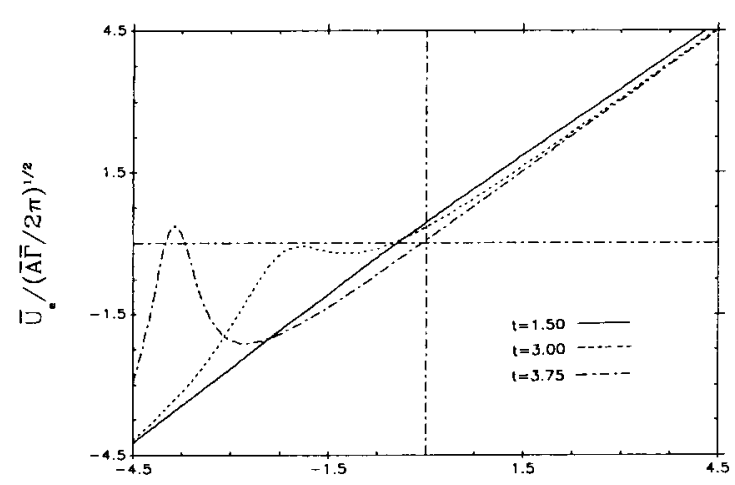

(b) EXTERNAL VELOCITY VERSUS $X$.

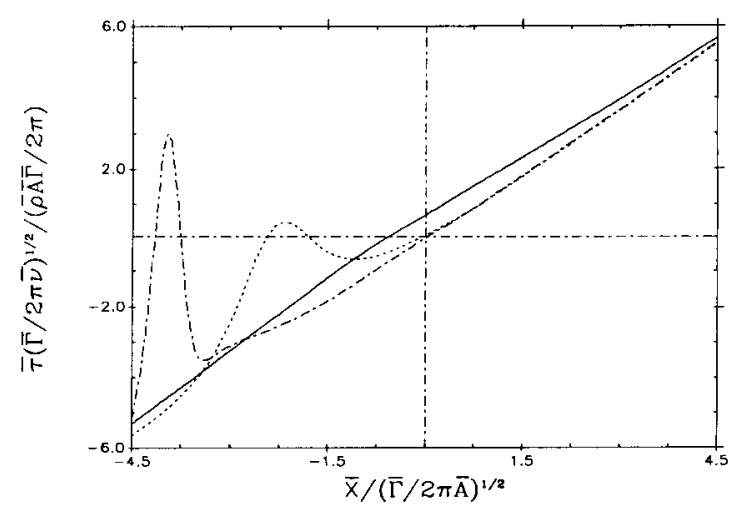

(c) WALL SHEAR VERSUS $X$.

FIGURE 6. - MOHENTUM SOLUTION. LEFT.
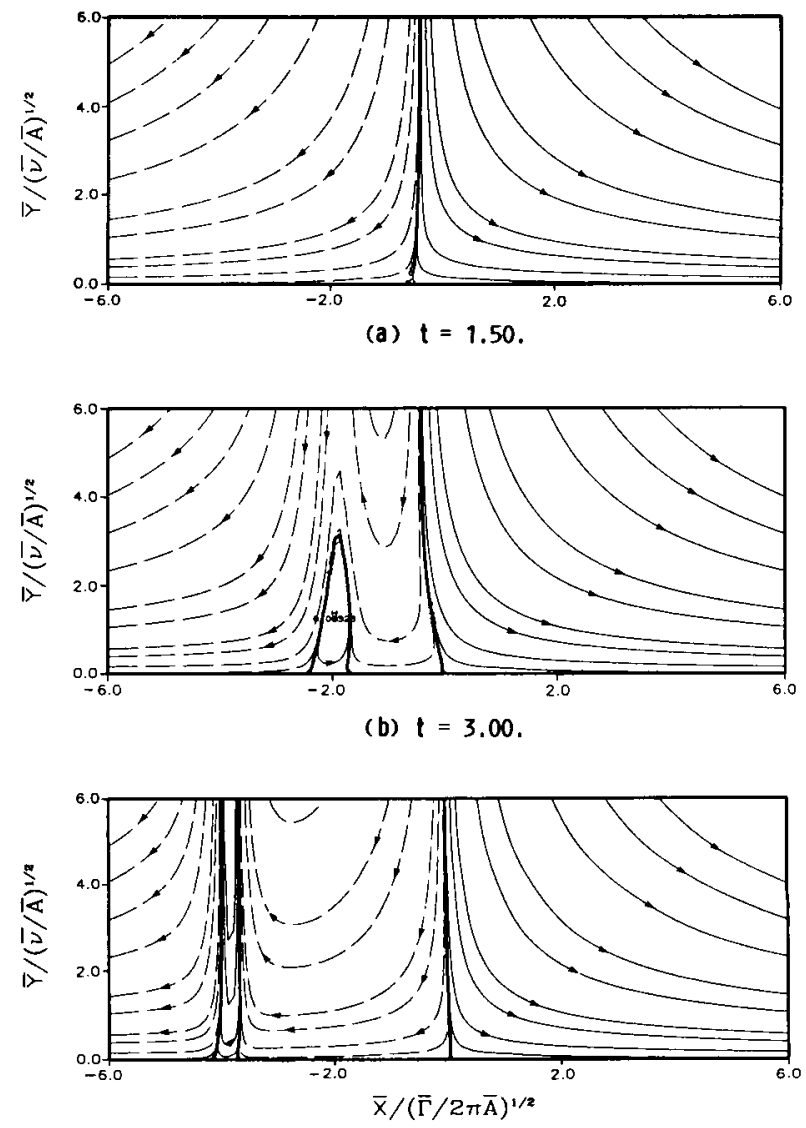

(c) $t=3.75$.

FIGURE 7. - STREANLINES, LEFT. 


\section{ORIGINAL PAGE IS \\ OF POOR QUALITY}

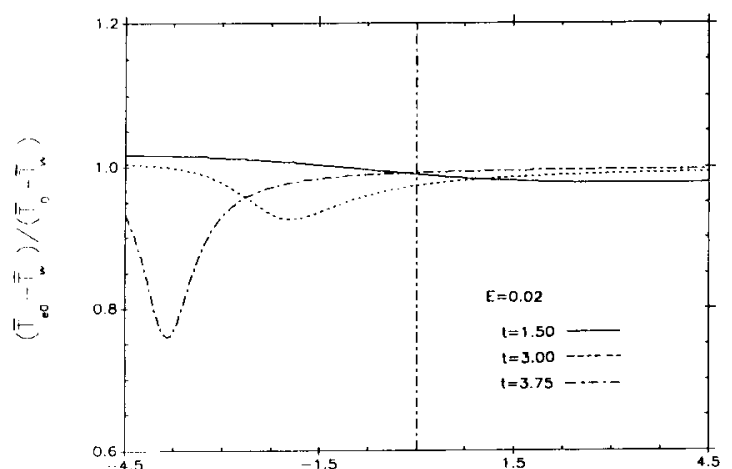

(a) EXTERNAL TOTAL TEMPERATURE VERSUS $X$.

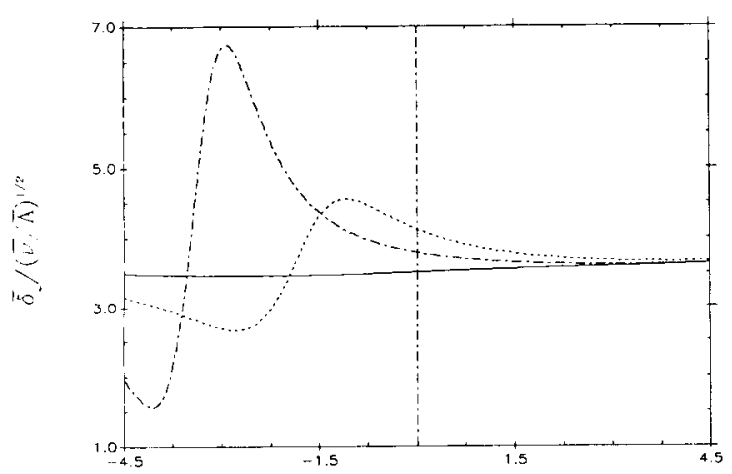

(b) DELTAT VERSUS $x$.

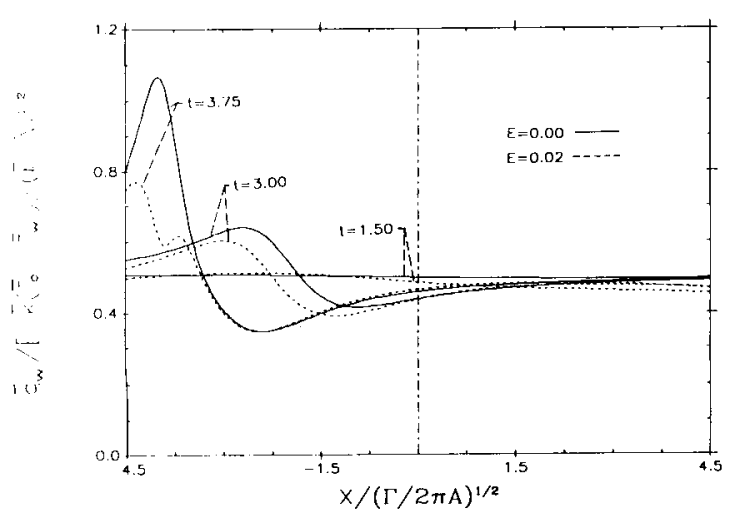

(c) WALL HEAT TRANSFER VERSUS $X$.

FIGURE 8. - ENERGY SOLUTION, LEFT.

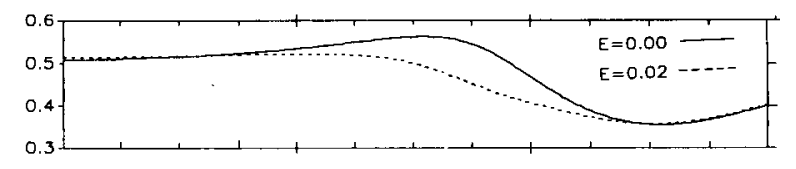

(a) $x=-2.0$.

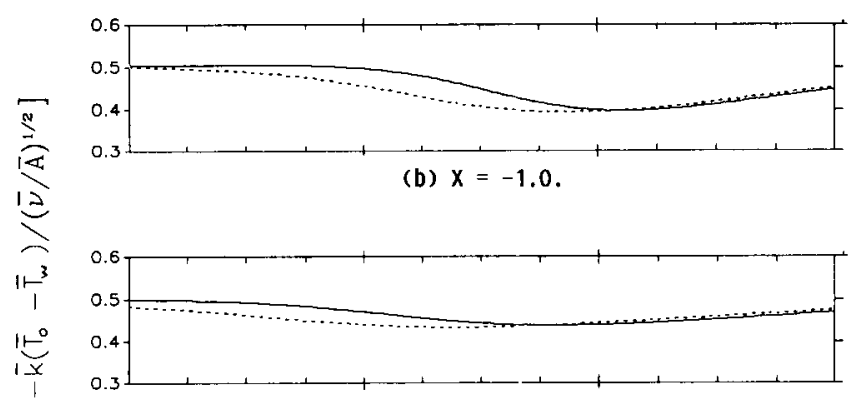

(c) $x=0.0$.

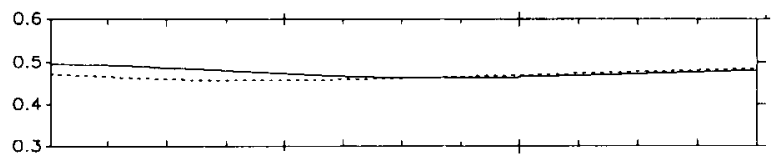

(d) $x=1.0$.

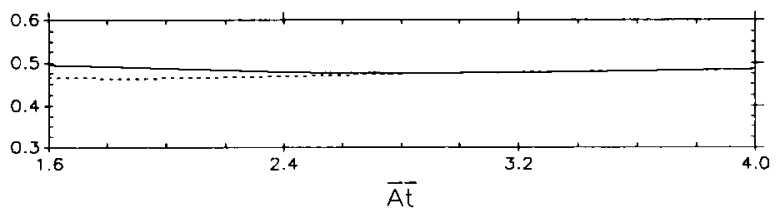

(e) $X=2.0$.

FiguRE 9. - WALL HEAT TRANSFER VERSUS $t$. LEFT. 


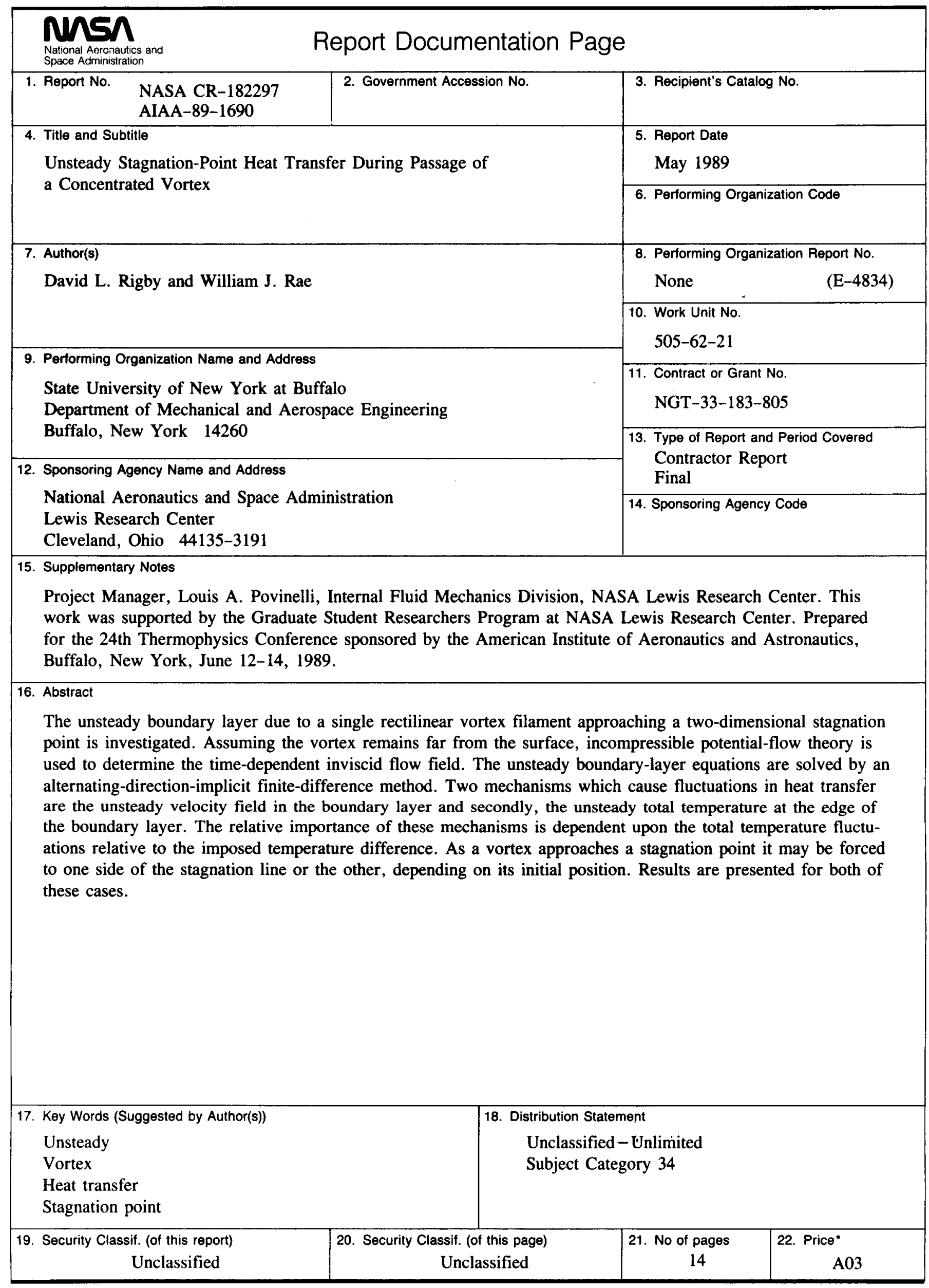

